\title{
The Dynamics of the Substructures and the Bulk Flow of the Local Group
}

\author{
V.G. Gurzadyan \\ Department of Theoretical Physics, Yerevan Physics Institute, Yerevan \\ 375036, Armenia. \\ S. Rauzy \\ Centre de Physique Théorique - C.N.R.S., Luminy Case 907, F-13288 \\ Marseille Cedex 9, France.
}

\begin{abstract}
The S-tree method is used to reveal the substructures of the Local Group, namely the membership of each individual galaxy in the Milky Way and M31 subgroups; the method is determining the degree of mutual influence of the galaxies. Once the main physical subgroups of the Local Group are defined, we use a general method of reconstruction of their bulk flow using the radial velocity distribution of the member galaxies. The results particularly indicate the essential mutual bulk flow of the Milky Way and M31 subgroups which does not simply coincide with the radial approaching velocity (blueshift) of M31. Finally, having obtained the $3 \mathrm{D}$ velocity vectors of both the subgroups, we determine the 3D bulk flow of the Local Group itself, which in the Cosmic Microwave Background rest frame is equal to $\mathrm{V}_{\mathrm{LG} \rightarrow \mathrm{CMB}}=668 \mathrm{~km} \mathrm{~s}^{-1}$ towards $l=$ 265 and $b=42$.
\end{abstract}

\section{Introduction}

The study of the properties of the substructures of the Local Group (LG) and, particularly of the 3D peculiar motions, are crucial for the understanding of its past and future evolution.

In the present study we consider the possibility to estimate the peculiar velocity of the LG using not the extragalactic information, but its internal dynamical properties combined with data on the CMB dipole. Namely, we analyse the substructure of the LG, and perform a procedure of $3 \mathrm{D}$ reconstruction of the velocity of the whole system and its main subgroups, using the method developed by Gurzadyan \& Rauzy (1997). The first step is performed by the S-tree technique (see Gurzadyan \& Kocharyan 1994) enabling, in particular, the analysis of systems such as groups or clusters of galaxies. For the second step, we use the data on the distribution of the line-of-sight velocities of the galaxies which are members of the LG and its subsystems, as revealed at the first step. Thus, we obtain the peculiar motions of the Milky Way and M31 subsystems with respect to each other. By the third step, from the bulk motions of the 
mentioned both subsystems we obtain the velocity vector of the Local Group in the CMB frame.

\section{The S-tree Technique}

The S-tree technique is developed for the investigation of properties of manydimensional nonlinear systems and essentially uses the concepts of the theory of dynamical systems (Gurzadyan et al. 1994; Gurzadyan \& Kocharyan 1994). Its idea is based on the property of structural stability well known in theory of dynamical systems, enabling study of the robust properties of the systems with a limited amount of information. The advantage of the method in the context of galaxy clusters is in the self-consistent use of both kinematical and positional information on the galaxies, as well as of the data on their magnitudes.

This approach is introducing the concept of the degree of boundness between the members of the given $\mathrm{N}$-body system, i.e. the definition of a non-negative function $\mathcal{P}$ called the boundness function which describes the degree of interaction of a given subset $Y$ of the initial set $\mathcal{A}$ with another subset $X \backslash Y$ according to some criterion. Particularly one can define a function

$$
\mathcal{P}: \mathcal{S}(\mathcal{A}) \rightarrow R_{+}:(X, Y) \mapsto \mathcal{P}_{X}(Y)
$$

where $\mathcal{S}(\mathcal{A})$ is the set of all subsets of the initial set, when one of the sets contains the other one. The procedure of such splitting of $\mathcal{A}$ can be measured by a nonnegative real number $\rho$ using the boundness function, so that the problem is reduced to the finding out of a function $\Sigma(\rho)$ denoting the set of all possible $\rho$-subsystems $\left\{\mathcal{A}_{1}, \ldots, \mathcal{A}_{d}\right\}$ of $(\mathcal{A}, \mathcal{P})$.

Attributing for the given $\rho$ the boundness matrix $D$ to another graph matrix $\Gamma$, the problem of the search of a $\rho$-bound cluster is reduced to that of the connected parts of the graph $\Gamma(\rho)$ - an $S$-tree diagram.

For the matrix $D_{a b}$ a representation via the two-dimensional curvature $K$ of the phase space of the system can be used

$$
D_{a b}=\max _{i, j}\left\{-K_{\nu}^{\mu}, 0\right\}
$$

where $\mu=(a, i) ; \nu=(b, j) ; a, b=1, \ldots, N ; i, j=1, \ldots, 3$. The two-dimensional curvature is represented by the Riemann tensor $R$ via the expression: $K_{\mu \nu}=$ $R_{\mu \nu \lambda \rho} u^{\lambda} u^{\rho}$. As a result, the degree of boundness between the members and subgroups of the given $\mathrm{N}$-body system can be obtained, thus revealing the physically interacting system (cluster) and its hierarchical substructure.

The computer code based on the S-tree method has been used for the study, for example, of the substructure of Abell clusters from the ENACS program (Gurzadyan \& Mazure 1998).

\section{Bulk Flow Reconstruction}

The reconstruction of the $3 \mathrm{D}$ velocity distribution function from the observed line-of-sight velocity distribution is an interesting inverse problem solved by Ambartsumian (1936) for stellar systems. The only assumption made was the 
independence of the distribution function on the spatial directions, or equivalently, that the $3 \mathrm{D}$ velocity distribution $\phi\left(v_{1}, v_{2}, v_{3}\right) d v_{1} d v_{2} d v_{3}$ of such systems was invariant under spatial translations, i.e. the probability density reads

$$
d P_{\mathrm{th}}=\phi\left(v_{1}, v_{2}, v_{3}\right) d v_{1} d v_{2} d v_{3} \rho(r, l, b) r^{2} \cos b d r d l d b
$$

where $\phi\left(v_{1}, v_{2}, v_{3}\right)$ is the $3 \mathrm{D}$ velocity distribution function (in galactic Cartesian coordinates) and $\rho(r, l, b)$ is the $3 \mathrm{D}$ spatial distribution function (in galactic coordinates). Under this assumption, Ambartsumian had proven the theoretical possibility of reconstructing of $\phi\left(v_{1}, v_{2}, v_{3}\right)$ from the observed radial velocity distribution function $f\left(v_{r}, l, b\right)$

$$
d P_{\mathrm{obs}}=f\left(v_{r}, l, b\right) \cos b d l d b d v_{r}
$$

where $v_{r}=\mathbf{v} \cdot \hat{\mathbf{r}}$ is the projection of the velocity $\mathbf{v}=\left(v_{1}, v_{2}, v_{3}\right)$ on the line-ofsight direction $\hat{\mathbf{r}}=\left(\hat{r}_{1}, \hat{r}_{2}, \hat{r}_{3}\right)=(\cos l \cos b, \sin l \cos b, \sin b)$.

However, computer experiments show that the direct application of Ambartsumian's formula is hardly possible for this purpose, because of the small number of galaxies in the system. Nevertheless, some quantities of interest such as the first order moment of this distribution, i.e. the mean $3 \mathrm{D}$ velocity of the system, can be obtained. Additional hypotheses on the distribution function $\phi\left(v_{1}, v_{2}, v_{3}\right)$ are then necessary. Hereafter, we assume that $\phi\left(v_{1}, v_{2}, v_{3}\right)$ can be written as follows

$$
\phi\left(v_{1}, v_{2}, v_{3}\right) d v_{1} d v_{2} d v_{3}=\prod_{i=1}^{3} \phi_{i}\left(v_{i}\right) d v_{i}
$$

where the distribution functions $\phi_{i}$ are centered on $\bar{v}_{i}$ and of variances $\sigma_{i}\left(\phi_{i}\left(v_{i}\right)\right.$ $\left.=\phi_{i}\left(v_{i} ; \bar{v}_{i}, \sigma_{i}\right)\right)$. We choose the Gaussian representation $\left(\phi_{1}=\phi_{2}=\phi_{3}=g\right.$ with $g$ Gaussian) and assume the isotropic velocity dispersions $\left(\sigma_{1}=\sigma_{2}=\sigma_{3}=\right.$ $\sigma_{v}$ ). The velocity field of the system is, thus, split into a mean $3 \mathrm{D}$ velocity $\mathbf{v}_{B}=\left(v_{x}, v_{y}, v_{z}\right)=\left(\bar{v}_{1}, \bar{v}_{2}, \bar{v}_{3}\right)$ (i.e. a bulk flow) plus a $3 \mathrm{D}$ random component, isotropic Maxwellian, centered on 0 and of velocity dispersion $\sigma_{v}$.

The fact that gravitating N-body systems do possess strong statistical properties peculiar to Kolmogorov systems (Gurzadyan \& Savvidy 1986; see also Gurzadyan \& Pfenniger 1994), is the justification for the separable form of distribution functions in Eqs.(3) and (5).

\section{The Local Group Subsystems.}

The S-tree analysis has revealed the following substructure of the LG (Gurzadyan et al. (1993)):

- Milky Way subsystem - 12 galaxies: Milky Way, SMC, Sculptor S, IC 1613, Fornax D, LMC, Carina, Leo I, Leo II, UM, Draco, N 6822;

- M31 subsystem - 7 galaxies: N 147, N 185, N 205, M31, M32, LGS-3, M33;

- The field galaxies of the Local Group - 13 galaxies: IC 10, N 55, IC 342, Leo A, N 3109, Sex A, DDO 155, Sagittarius, DDO 210, IC 5152, Hog, Pegasus, WLM.

Thus the membership of galaxies in two main subgroups - dominated by the Milky Way and M31 - has been revealed, in general confirming the conventional views. Physical connections between some individual galaxies have been 
also indicated, not reported before (e.g. NGC 6822 and IC 1613). Some galaxies appeared to have no actual influence on the dynamics of LG and vice versa, and therefore, were considered not to be its members. The existence of galaxies obscured by the Galactic disk, like the newly-discovered Dwingeloo 1 and 2, also cannot be ruled out, though it seems unlikely that at least the latter ones can essentially influence the substructure of the LG (Lynden-Bell 1996, private communication). Thus in accord with our analysis, the MW and M31 subsystems contain respectively 12 and 7 galaxies.

For each subsystem, as assumed in Eq. (3), the velocity distribution function $\phi\left(v_{1}, v_{2}, v_{3}\right)$ is translation invariant, so that $\phi\left(v_{1}, v_{2}, v_{3}\right)$ can be split into a bulk flow $\mathbf{v}_{B}=\left(v_{x}, v_{y}, v_{z}\right)$ plus a $3 \mathrm{D}$ random component, isotropic Maxwellian, centered on 0 and of velocity dispersion $\sigma_{v}$. The standard bulk flow statistics can be derived by means of the likelihood function, i.e. $\mathcal{L}=\mathcal{L}\left(v_{x}, v_{y}, v_{z}, \sigma_{v}\right)$ which reads thus :

$$
\mathcal{L}=-\ln \sigma_{v}-\frac{1}{N} \sum_{k=1}^{N} \frac{\left(v_{r}^{k}-v_{x} \hat{r}_{1}^{k}-v_{y} \hat{r}_{2}^{k}-v_{z} \hat{r}_{3}^{k}\right)^{2}}{2 \sigma_{v}^{2}}
$$

where $\hat{r}_{1}^{k}=\cos l^{k} \cos b^{k}, \hat{r}_{2}^{k}=\sin l^{k} \cos b^{k}$ and $\hat{r}_{3}^{k}=\sin b^{k} ; k$ denotes the k-th galaxy. Maximizing $\mathcal{L}$ with respect to $v_{x}, v_{y}$ and $v_{z}$ leads to the $3 \times 3$ matrix equation

$$
\left[\begin{array}{l}
v_{x} \\
v_{y} \\
v_{z}
\end{array}\right]=\mathbf{M}^{-1}\left[\begin{array}{c}
\left\langle v_{r} \hat{r}_{1}\right\rangle \\
\left\langle v_{r} \hat{r}_{2}\right\rangle \\
\left\langle v_{r} \hat{r}_{3}\right\rangle
\end{array}\right]
$$

where

$$
\mathbf{M}=\left[\begin{array}{lll}
\left\langle\hat{r}_{1} \hat{r}_{1}\right\rangle & \left\langle\hat{r}_{1} \hat{r}_{2}\right\rangle & \left\langle\hat{r}_{1} \hat{r}_{3}\right\rangle \\
\left\langle\hat{r}_{1} \hat{r}_{2}\right\rangle & \left\langle\hat{r}_{2} \hat{r}_{2}\right\rangle & \left\langle\hat{r}_{2} \hat{r}_{3}\right\rangle \\
\left\langle\hat{r}_{1} \hat{r}_{3}\right\rangle & \left\langle\hat{r}_{2} \hat{r}_{3}\right\rangle & \left\langle\hat{r}_{3} \hat{r}_{3}\right\rangle
\end{array}\right]
$$

where $\langle$.$\rangle denotes the average on the sample.$

The results of computations are as follows:

- Milky Way subsystem: $\left(v_{x}=104 \pm 64, v_{y}=-116 \pm 42, v_{z}=45 \pm 33\right)$ or $\mathbf{v}_{B}=$ $162 \mathrm{~km} \mathrm{~s}^{-1}$ in the direction $(l=312, b=16)$ or $(\alpha=1338.75, \delta=-4543.8)$ with a velocity dispersion of $\sigma_{v}=69 \pm 15 \mathrm{~km} \mathrm{~s}^{-1}$;

- M31 subsystem: $\left(v_{x}=-117 \pm 541, v_{y}=85 \pm 267, v_{z}=309 \pm 254\right)$ or $\mathbf{v}_{B}=341$ $\mathrm{km} \mathrm{s}^{-1}$ in the direction $(l=144, b=65)$ or $(\alpha=1154.35, \delta=5007.09)$ with a velocity dispersion $\sigma_{v}=69 \pm 15 \mathrm{~km} \mathrm{~s}^{-1}$.

These velocities are expressed in $\mathrm{km} \mathrm{s}^{-1}$ and with respect to the rest frame of the Milky Way. For each system, the values of the velocity dispersion, $\sigma_{v}$, and of the accuracy of the bulk flow estimate, are calculated by using numerical simulations (see Rauzy \& Gurzadyan 1998 for details).

In the coordinate frame such that the X-axis is aligned with the M31 line-ofsight (i.e., toward $l=121.2$ and $b=-21.6$ ) and $\mathrm{Y}$-axis lies in the Galactic plane, the $3 \mathrm{D}$ bulk flow of the M31 subgroup reads $\mathbf{v}_{B}=(10 \pm 34,72 \pm 623,335 \pm 291)$. In these coordinates, the bulk flow of the MW subsystem reads $\mathbf{v}_{B}=(-155 \pm$ $57,-26 \pm 53,-15 \pm 26)$.

To check this, we have repeated our analysis on the 32 Local Group galaxies situated nearer than $2 \mathrm{Mpc}$, but ignoring the MW and M31 dynamical substructures. The values of the velocity dispersion estimator for the MW and M31 
subsamples appear to be significantly greater than the above mentioned ones, thus indicating the existence of genuine kinematic substructures in the Local Group, and so strengthening the present analysis.

\section{Discussion}

The motions of the two subsystems of the Local Group have been estimated, containing respectively 7 and 12 galaxies, as identified via the $\mathrm{S}$-tree technique.

We have obtained that the relative velocity of the M31 subsystem with respect to the MW subsystem, when projected on the line joining MW to M31, is $-165 \pm 66 \mathrm{~km} \mathrm{~s}^{-1}$, confirming the conventional views (e.g., Peebles 1994). On the other hand, the 3D inner motions inside the Local Group, if significant, are found to be surprisingly large: the M31 subsystem has a relative 3D velocity of amplitude $399 \mathrm{~km} \mathrm{~s}^{-1}$ with respect to the MW subsystem.

The CMB temperature dipole, if interpreted as the signature of our motion with respect to the rest frame of this radiation (CMB frame), gives for the Milky Way a peculiar velocity $\mathrm{V}_{\mathrm{MW} \rightarrow \mathrm{CMB}}$ of $552 \mathrm{~km} \mathrm{~s}^{-1}$ pointing toward the galactic coordinates $l=266$ and $b=29$, e.g., Kogut et al. (1993). This MW motion in the CMB frame is traditionally split into two components

$$
\mathrm{V}_{\mathrm{MW} \rightarrow \mathrm{CMB}}=\mathrm{V}_{\mathrm{MW} \rightarrow \mathrm{LG}}+\mathrm{V}_{\mathrm{LG} \rightarrow \mathrm{CMB}}
$$

where $\mathrm{V}_{\mathrm{MW} \rightarrow \mathrm{LG}}$ is the velocity of the Milky Way relative to Local Group rest frame, which originates from the internal non-linear dynamics governing the Local Group and $\mathrm{V}_{\mathrm{LG} \rightarrow \mathrm{CMB}}$ is the peculiar motion of the Local Group as a whole in the CMB frame, created by large scale mass fluctuations present in the Universe. The latter can be inferred in some sense from various dipoles obtained up to now, such as the optical galaxies, X-ray galaxies, IRAS galaxies, clusters of galaxies, etc. (see Branchini \& Plionis 1996).

Note that the peculiar motion of the Milky Way in the Local Group rest frame $\mathrm{V}_{\mathrm{MW} \rightarrow \mathrm{LG}}$ can be obtained from the analysis of the dynamics of the Local Group and its substructures. Subtracting it from the observed $V_{M W} \rightarrow C M B$ thus gives a local estimate of $\mathrm{V}_{\mathrm{LG} \rightarrow \mathrm{CMB}}$, which can be compared with its values extracted from a dipole analysis, as mentioned above.

Herein, we assign to the Local Group the mean motion of its main substructures, i.e. both MW and M31 subsystems being equally weighted. It gives $\mathrm{V}_{\mathrm{MW} \rightarrow \mathrm{LG}}=(7 \pm 303,15 \pm 155,-177 \pm 144)$ or $178 \mathrm{~km} \mathrm{~s}^{-1}$ toward $l=65$ and $b=-85$. Finally, our local estimate of the LG peculiar velocity in the CMB frame yields $\mathrm{V}_{\mathrm{LG} \rightarrow \mathrm{CMB}}=(-41 \pm 303,-497 \pm 155,445 \pm 144)$ or $668 \mathrm{~km} \mathrm{~s}^{-1}$ in amplitude pointing toward $l=265$ and $b=42$. This result within $1 \sigma$ level is in agreement with the estimation by Yahil et al. (1977), based on the Solar system motion relative to the LG centroid : $\mathrm{V}_{\mathrm{LG} \rightarrow \mathrm{CMB}}=622 \mathrm{~km} \mathrm{~s}^{-1}$ toward $l=277$ and $b=30$ or $(66,-535,311)$ in galactic Cartesian coordinates.

The existence of some discrepancy between these two values, if significant, can be interpreted as follows. The LG centroid had been defined by specific choice of the main and satellite populations and with further search of the bestfit solution for the Solar system motion. 
The fact of existence of an essential bulk flow of the substructures revealed above, is a remarkable property of the LG and can be a common feature of subgroups of clusters of galaxies (Gurzadyan \& Mazure 1998) - galaxy associations.

Acknowledgements. We thank Donald Lynden-Bell for valuable discussions. During this study VG was supported by Université de la Méditérannée within the French-Armenian collaboration program. VG is thankful also to the organizers who made possible the participation in the symposium.

\section{References}

Ambartzumian, V.A. 1936, MNRAS, 96, 172

Branchini, E., Plionis, M. 1996, ApJ, 460, 569

Gurzadyan, V.G., Kocharyan, A.A. 1994, in: Paradigms of the Large-Scale Universe, Gordon and Breach

Gurzadyan, V.G., Harutyunyan, V.V., Kocharyan, A.A. 1994, A\&A, 281, 964

Gurzadyan, V.G., Kocharyan, A.A., Petrosian, A.R. 1993, Ap\&SS, 201, 243

Gurzadyan, V.G., Mazure, A. 1998, MNRAS, 295, 177

Gurzadyan, V.G., Pfenniger, D. (eds.) 1994, Ergodic Concepts in Stellar Dynamics, Lecture Notes in Physics, vol. 430, Springer-Verlag.

Gurzadyan, V.G., Rauzy, S. 1997, Astrophysics, 40, 308

Gurzadyan, V.G., Savvidy, G.K. 1986, A\&A, 160, 203

Kogut, A., et al. 1993, ApJ, 419, 1

Peebles, P.J.E. 1994, ApJ, 429, 43

Rauzy, S., Gurzadyan, V.G. 1998, MNRAS, 298, 114

Yahil, A., Tammann, G.A., Sandage, A. 1977, ApJ, 217, 903 